\title{
Laurence-Moon-Bardet-Biedl Syndrome
}

\author{
Sahu JK,' Jain $V^{1}$ \\ 'Department of Pediatrics, All India Institute of Medical Sciences, New Delhi, India
}

\begin{abstract}
Laurence-Moon-Bardet-Biedl syndrome is a rare, genetically heterogeneous, autosomal recessive inherited disorder with wide variability in expression. We report a case of Laurence-Moon-BardetBiedl syndrome with typical phenotype in conjunction with nonalcoholic steatohepatitis. The diagnosis had been missed until the patient presented at our hospital.
\end{abstract}

Key words: laurence-moon-bardet-biedl syndrome, obesity, polydactyly, retinitis pigmentosa

\section{INTRODUCTION}

Laurence-Moon-Bardet-Biedl syndrome (LMBBS) is a rare, genetically heterogeneous, autosomal recessive disorder characterized by early onset retinitis pigmentosa, post axial polydactyly, central obesity, mental retardation, hypogonadism and kidney structural abnormalities or functional impairment. ${ }^{1}$ We report here a typical case of Laurence-Moon-Bardet-Biedl syndrome in an eleven-year old boy with additional finding of $\mathrm{NASH}$. We also present a review of the diagnosis and management of this rare and interesting syndrome.

\section{CASE REPORT}

An eleven year Muslim boy was brought to the Pediatric Endocrinology Clinic of our hospital with complaint of excessive weight gain noticed since early childhood. He was born out of non-consanguineous marriage of a 29 year-old mother and 34 year old father. He was born at term, with no antenatal or perinatal complications. The patient was fourth of seven bothers and sister, with all other family members being normal. He had progressive deterioration in vision for the last five to six years, starting with difficulty in seeing at night.
There was history of development delay also. He had consulted many pediatricians and ophthalmologists for his condition but remained undiagnosed and presented to our tertiary care hospital.

On examination, he was markedly obese. His weight was $62 \mathrm{~kg}(+3.6 \mathrm{SDS})$, height was $152 \mathrm{~cm}$ (+1SDS) and $\mathrm{BMI}$ was $26.8 \mathrm{~kg} / \mathrm{m}^{2}(+3.2 \mathrm{SDS})$. His waist circumference was $92.5 \mathrm{~cm}$. His blood pressure was $118 / 70 \mathrm{~mm} \mathrm{Hg}$. There was postaxial polydactyly of all four limbs (Figure1a and b). Examination of the external genitalia revealed stretched penile length $3 \mathrm{~cm}$ (microphallus) and testicular volume of $3 \mathrm{ml}$ bilaterally. Ophthalmologic examination revealed visual acuity of $1 / 60$ (right) and 2/60 (left). Fundus showed a waxy pale disc with marked attenuation of arterioles and pigment clumps at the periphery (Figure 2) suggestive of retinitis pigmentosa. His 10 was 55 .

The fasting blood glucose was $84 \mathrm{mg} \%$. Renal function tests were normal. Liver function tests showed elevated enzymes with an SGOT of 83 IU and SGPT of $132 \mathrm{IU}$. Viral markers for hepatitis $B$ and $C$ were negative. The lipid profile was as follows: Total cholesterol 173mg\%, H.D.L.: $41 \mathrm{mg} \%$, V.L.D.L.: $23 \mathrm{mg} \%$, L.D.L.: 109mg\%,

\author{
Correspondence: \\ Dr. Vandana Jain \\ Department of Pediatrics \\ All India Institute of Medical Sciences \\ New Delhi - 110029 \\ Email: drvandanajain@gmail.com
}


Triglycerides $117 \mathrm{mg} \%$; all of which are within normal limits. His hormone profile was FSH $3.31 \mathrm{miu} / \mathrm{ml}$ (normal1-8), LH 0.30miu/ml (normal 2-12), Testosterone $0.12 \mathrm{nmol} / \mathrm{l}$ (normal 8.71-36.47), TSH 3.77 micro iu/ $\mathrm{ml}$ (normal 0.49-4.67). Urine examination revealed +1 proteinuria. A 24-h urinary collection revealed $0.2 \mathrm{~g}$ of protein. Ultrasound scan of abdomen showed fatty, enlarged liver with normal kidneys.

A diagnosis of Laurence-Moon-Bardet-Biedl syndrome having central obesity, postaxial polydactyly, mental retardation, retinitis pigmentosa, hypogonadism and functional renal impairment was made. An additional finding in this case was nonalcoholic steatohepatitis (NASH) as diagnosed by enlarged fatty liver on sonography with elevated liver enzymes. He was prescribed glasses, advised for fat and refined carbohydrate-restricted diet and exercise and asked for follow-up after 3 months. He will specifically need to be followed up for pubertal development for the next few years as hypogonadism is a likely problem.

\section{DISCUSSION}

The patient above is a classical case of LaurenceMoon-Bardet-Biedl syndrome. Steatohepatitis was an additional feature that occurs in $18.5 \%$ of markedly obese patients. ${ }^{2}$

The syndrome was independently described by Bardet and Biedl in the 1920s. There was controversy in medical literature with the condition described in 1865 by Laurence and Moon, referred as Laurence and Moon syndrome (LMS). After a 22-year prospective cohort study of 46 patients from 26 Newfoundland families with BBS, Moore et al concluded that BBS and LMS are different spectrum of same entity. ${ }^{3}$ The variable manifestations of this syndrome can be explained at molecular basis by ciliopathy.

The condition was thought to be rare, but this may have been due to the failure to diagnose incomplete or partial cases. The prevalence of LMBBS is $1: 160000$ in Europe. However, its prevalence is markedly increased in highly consanguineous Arab-Bedouin communities in the Arab population of Kuwait (1: 13500$).{ }^{4}$

Recent advances in genetics have enabled investigators to define syndromes by specific mutations. Eleven genes are known to be associated with this syndrome: BBS1, BBS2, ARL6/BBS3, BBS4, BBS5, MKKS/BBS6, BBS7, TTC8/BBS8, B1/BBS9, BBS10, and TRIM32/ BBS1 1.4 The syndrome is transmitted as an autosomal recessive trait. There is considerable heterogeneity and intrafamilial variation in the extent and severity of clinical manifestations of LMBBS. The diagnosis of LMBBS is established by clinical criteria suggested by Beales et al that the presence of four primary features or three primary features plus two secondary features

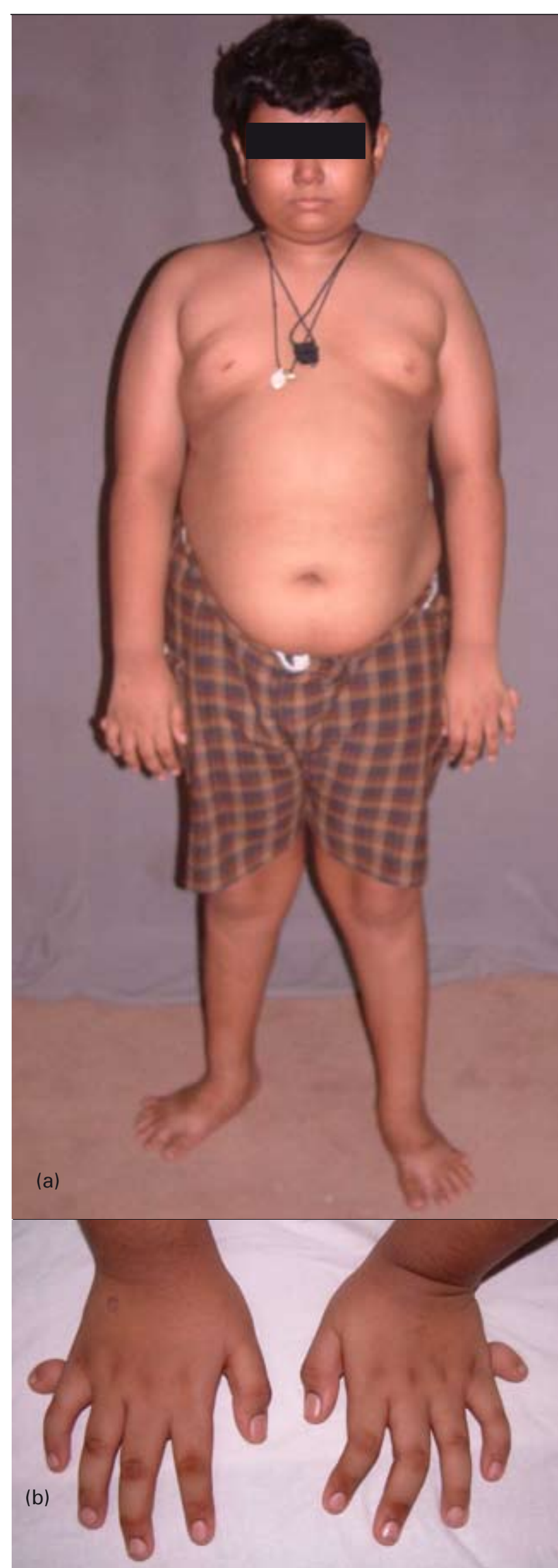

Figure 1(a \& b). Obese child with postaxial polydactyly of all four limbs. 
Sahu et al. Laurence-Moon-Bardet-Biedl Syndrome

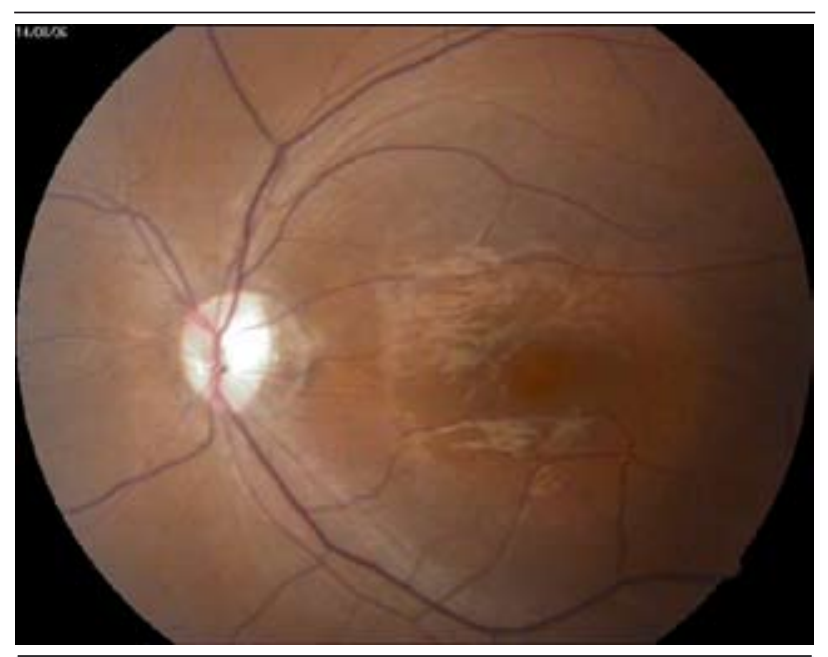

Figure 2. Fundus of left eye showing retinitis pigmentosa.

is diagnostic. ${ }^{5}$ Primary features include rod-cone dystrophy, polydactyly, obesity, learning disabilities, hypogonadism in males and renal anomalies. Secondary features include speech disorder, brachydactyly, developmental delay, polyuria/polydipsia, ataxia, poor coordination/clumsiness, left ventricular hypertrophy, diabetes mellitus, hepatic fibrosis and spasticity.

Central obesity is the commonest feature of LMBBS. It develops in early childhood and is aggravated with age. Ocular manifestations are also common and become apparent between the ages of 4 and 10 years. They include retinal dystrophic changes which progresses relentlessly leading to decreased night vision, reduced visual acuity and blindness at a young age. Mild to moderate mental retardation is an additional feature of the syndrome. Hypogonadism in affected males is common. Dysmorphism of extremities, including postaxial polydactyly, syndactyly or brachydactyly, is one of the earliest and most common manifestations of LMBBS. Renal involvement is observed in most affected individuals. It consists of structural and functional abnormalities such as calyceal or pelvic dilatation, fetal lobulation, and focal and diffuse cortical loss, as well as tubular dysfunction, hypertension and progressive renal failure. ${ }^{6}$ Many children with LMBBS are delayed in reaching major developmental milestones and speech disorder has been reported infrequently in this syndrome.

LMBBS has an adverse prognosis, with early onset of blindness, obesity, hypertension, and diabetes mellitus. Renal impairment is frequent often goes undetected. This is significant in that early death often occurs in this condition because of the renal disease. Their survival and quality of life depend on the severity of clinical features, as well as on the quality of the medical care they receive.

LMBBS with visual impairment need visual aids and educational programs. For management of obesity, diet, exercise and behavioral therapies are advocated. Accessory digits may be removed surgically to prevent functional interference and poor fitting of footwear. Early intervention and special education is needed to address cognitive disability. Speech delay/ impairment needs early diagnosis and speech therapy. Hormone replacement therapy is advocated to correct hypogonadism. Renal anomalies and hypertension are treated as in the other affected children. Surveillance includes regular ophthalmologic evaluation, annual blood pressure measurement, monitoring of renal function, and regular testing for diabetes mellitus and lipid profile. These concerns are best addressed in the setting of a dedicated team made up of the appropriate specialties allowing proper planning and cooperation so that the patient may receive the best possible care. The main purpose of this case report, besides reporting a very rare entity was to make the reader aware of management strategies and adverse prognosis. The condition is supposed to be rare, but this may have been due to the failure to diagnose incomplete or partial cases. Pediatricians Ophthalmologists, endocrinologists and nephrologists should be aware of LMBBS because of its adverse prognosis.

\section{REFERENCES}

1. Schachat AP, Maumenee IH. The Bardet-Biedl syndrome and related disorders. Arch Ophthal1982;100:285-8.

2. Wanless IR, Lentz JS. Fatty liver hepatitis (steatohepatitis) and obesity: an autopsy study with analysis of risk factors. Hepatology 1990;12(5):1106-10.

3. Moore SJ, Green JS, Fan Y, Bhogal AK, Dicks E, Fernandez $\mathrm{BA}$, et al. Clinical and genetic epidemiology of BardetBiedl syndrome in Newfoundland: a 22-year prospective, population-based, cohortstudy. Am J Med Genet 2005;132:35260 .

4. Woods MO, Young TL, Parfrey PS, Hefferton D, Green JS, Davidson WS. Genetic heterogeneity of Bardet-Biedl syndrome in a distinct Canadian population: evidence for a fifth locus. Genomics1999;55:2-9.

5. Beales PL, Elcioglu N, Woolf AS, Parker D, Flinter FA. New criteria for improved diagnosis of Bardet-Biedl syndrome: results of a population survey. J Med Genet 1999;36(6):43746

6. O'Dea D, Parfrey PS, Harnett JD, Hefferton D, Cramer $\mathrm{BC}$, Green J. The importance of renal impairment in the natural history of Bardet-Biedl syndrome. Am J Kidney Dis 1996;27(6):776-83. 